PROCEEDINGS OF THE

AMERICAN MATHEMATICAL SOCIETY

Volume 131, Number 5, Pages 1405-1413

S 0002-9939(02)06896-X

Article electronically published on December 6, 2002

\title{
ON QUASI-AFFINE TRANSFORMS OF READ'S OPERATOR
}

\author{
THOMAS SCHLUMPRECHT AND VLADIMIR G. TROITSKY
}

(Communicated by David R. Larson)

\begin{abstract}
We show that C. J. Read's example of an operator $T$ on $\ell_{1}$ which does not have any non-trivial invariant subspaces is not the adjoint of an operator on a predual of $\ell_{1}$. Furthermore, we present a bounded diagonal operator $D$ such that even though $D^{-1}$ is unbounded, the operator $D^{-1} T D$ is a bounded operator on $\ell_{1}$ with invariant subspaces, and is adjoint to an operator on $c_{0}$.
\end{abstract}

\section{INTRODUCTION}

In this note we deal with the Invariant Subspace Problem, the problem of the existence of a closed non-trivial invariant subspace for a given bounded operator on a Banach space. The problem was solved in the positive for certain classes of operators (see [RR73, AAB98] for details), however in the mid-seventies P. Enflo Enf76, Enf87 constructed an example of a continuous operator on a Banach space with no invariant subspaces, thus answering the Invariant Subspace Problem for general Banach spaces in the negative. In Read85. C. J. Read presented an example of a bounded operator $T$ on $\ell_{1}$ with no invariant subspace. Recently V. Lomonosov suggested that every adjoint operator has an invariant subspace. In the first part of this note we show that the Read operator $T$ is not an adjoint of any bounded operator defined on some predual of $\ell_{1}$.

Suppose that $A$ has a non-trivial invariant (or a hyperinvariant) subspace, and suppose that $B$ is similar to $A$, that is, $B=C A C^{-1}$ for some invertible operator $C$. Clearly, $B$ also has a non-trivial invariant (respectively hyperinvariant) subspace. Moreover, it is known (see [RR73, Theorem 6.19]) that if $A$ has a hyperinvariant subspace and $B$ is quasi-similar to $A$ (that is, $C A=B C$ and $A D=D B$, where $C$ and $D$ are two bounded one-to-one operators with dense range), then $B$ also has a hyperinvariant subspace. To our knowledge it is still unknown whether or not $A$ has a non-trivial invariant subspace if and only if $B$ has a non-trivial invariant subspace, assuming $A$ and $B$ are quasi-similar.

Recall (cf. [Sz-NF68]) that an operator $A$ is said to be a a quasi-affine transform of $B$ if $C A=B C$, for some injective operator $C$ with dense range. In the second part of this paper we construct an injective diagonal operator $D$ on $\ell_{1}$ such that even though $D^{-1}$ is unbounded, the operator $S=D^{-1} T D$ ( $T$ being Read's operator)

Received by the editors November 30, 2001.

2000 Mathematics Subject Classification. Primary 47A15; Secondary 47 B37.

The first author was supported by the NSF. Most of the work on the paper was done during the Workshop on linear analysis and probability at Texas A\&M University, College Station. 
is bounded and has an invariant subspace. Thus, we show that a quasi-affine transform of an operator with no non-trivial invariant subspace might have a nontrivial invariant subspace. Furthermore, $S$ is the adjoint of a bounded operator on $c_{0}$.

Although we prove our statement for a specific choice of $D$, it is true for a much more general choice, and it seems to be true for any diagonal operator $D$ that $S=D^{-1} T D$ has a non-trivial invariant subspace, whenever $S$ is an adjoint of an operator on $c_{0}$. More generally, the following question is of interest in view of the above-mentioned conjecture by $\mathrm{V}$. Lomonosov.

Question. Does every quasi-affine transform of Read's operator, which is an adjoint of an operator on $c_{0}$, have a non-trivial invariant subspace?

We introduce the following notations. Following Read86 we denote by $F$ the vector space of all eventually vanishing scalar sequences, and by $\left(f_{i}\right)$ the standard unit vector basis of $F$. For an $x=\sum a_{i} f_{i} \in F$, we define the support of $x$ to be the set $\left\{i \in \mathbb{N}: a_{i} \neq 0\right\}$ and denote it by $\operatorname{supp}(x)$. The linear span of some subset $A$ of a vector space is denoted by $\operatorname{lin} A$.

\section{READ'S OPERATOR IS NOT ADJOINT}

We begin by reminding the reader of the construction of the operator $T$ in Read85 Read86. It depends on a strictly increasing sequence $\mathbf{d}=\left(a_{1}, b_{1}, a_{2}, b_{2}, \ldots\right)$ of positive integers which has to be chosen to be sufficiently rapidly increasing. Also let $a_{0}=1, v_{0}=0$, and $v_{n}=n\left(a_{n}+b_{n}\right)$ for $n \geqslant 1$.

Read's operator $T$ is defined by prescribing the orbit $\left(e_{i}\right)_{i \geqslant 0}$ of the first basis element $f_{0}$.

Definition 2.1. There is a unique sequence $\left(e_{i}\right)_{i=0}^{\infty} \subset F$ with the following properties:

(0) $f_{0}=e_{0}$;

(A) if integers $r, n$, and $i$ satisfy $0<r \leqslant n, i \in\left[0, v_{n-r}\right]+r a_{n}$, we have

$$
f_{i}=a_{n-r}\left(e_{i}-e_{i-r a_{n}}\right) ;
$$

(B) if integers $r, n$, and $i$ satisfy $1 \leqslant r<n, i \in\left(r a_{n}+v_{n-r},(r+1) a_{n}\right)$, (respectively, $1 \leqslant n, i \in\left(v_{n-1}, a_{n}\right)$ ), then

$$
\left.f_{i}=2^{(h-i) / \sqrt{a_{n}}} e_{i} \text {, where } h=\left(r+\frac{1}{2}\right) a_{n} \text { (respectively, } h=\frac{1}{2} a_{n}\right) ;
$$

(C) if integers $r, n$, and $i$ satisfy $1 \leqslant r \leqslant n, i \in\left[r\left(a_{n}+b_{n}\right), n a_{n}+r b_{n}\right]$, then

$$
f_{i}=e_{i}-b_{n} e_{i-b_{n}}
$$

(D) if integers $r, n$, and $i$ satisfy $0 \leqslant r<n, i \in\left(n a_{n}+r b_{n},(r+1)\left(a_{n}+b_{n}\right)\right)$, then

$$
f_{i}=2^{(h-i) / \sqrt{b_{n}}} e_{i}, \text { where } h=\left(r+\frac{1}{2}\right) b_{n} .
$$

Indeed, since $f_{i}=\sum_{j=0}^{i} \lambda_{i j} e_{j}$ for each $i \geqslant 0$ and $\lambda_{i i}$ is always nonzero, this linear relation is invertible. Further,

$$
\operatorname{lin}\left\{e_{i} \mid i=1, \ldots, n\right\}=\operatorname{lin}\left\{f_{i} \mid i=1, \ldots, n\right\} \text { for every } n \geqslant 0 .
$$

In particular, all $e_{i}$ are linearly independent and also span $F$. Then Read defines $T: F \rightarrow F$ to be the unique linear map such that $T e_{i}=e_{i+1}$. Read proves that $T$ can be extended to a bounded operator on $\ell_{1}$ with no invariant subspaces provided d increases sufficiently rapidly. 
Proposition 2.2. $T$ is not the adjoint of an operator $S: X \rightarrow X$ where $X$ is a Banach space whose dual is isometric to $\ell_{1}$.

Proof. Assume that our claim is not true. Then there is a local convex topology $\tau$ on $\ell_{1}$ so that

(a) $\tau$ is weaker than the norm topology of $\ell_{1}$;

(b) $\mathrm{B}\left(\ell_{1}\right)$ is sequentially compact with respect to $\tau$;

(c) if $\left(x_{n}\right) \subset \ell_{1}$ converges with respect to $\tau$ to $x$, then $\liminf _{n \rightarrow \infty}\left\|x_{n}\right\| \geqslant\|x\|$;

(d) $T$ is continuous with respect to $\tau$.

Note that with respect to any predual $X$ of $\ell_{1}$ the weak* topology has properties (a)-(d). Let $s \in \mathbb{N}$ be fixed, and $n>s$. Then $f_{(n-s) a_{n}}=a_{s}\left(e_{(n-s) a_{n}}-e_{0}\right)$ by (A) above. It follows that $T^{v_{s}+1} f_{(n-s) a_{n}}=a_{s}\left(e_{(n-s) a_{n}+v_{s}+1}-e_{v_{s}+1}\right)$. Further, it follows from (B) that $e_{(n-s) a_{n}+v_{s}+1}$ equals $2^{\left(1+v_{s}-\frac{1}{2} a_{n}\right) / \sqrt{a_{n}}} f_{(n-s) a_{n}+v_{s}+1}$ and converges to zero in norm (and, hence, in $\tau$ ) as $n \rightarrow \infty$. Therefore

$$
\tau_{n \rightarrow \infty} \lim ^{v_{s}+1} f_{(n-s) a_{n}}=-a_{s} e_{v_{s}+1}=T^{v_{s}+1}\left(-a_{s} e_{0}\right) .
$$

Notice that $T^{v_{s}+1}$ is $\tau$-continuous and one-to-one because its null space is $T$ invariant. By sequential compactness of $B\left(\ell_{1}\right)$, the sequence $f_{(n-s) a_{n}}$ must have a $\tau$-convergent subsequence. Then, by (1), the limit point has to be $-a_{s} e_{0}$. Since that argument applies to any subsequence, we deduce that

$$
\tau \text { - } \lim _{n \rightarrow \infty} f_{(n-s) a_{n}}=-a_{s} e_{0} .
$$

Since $\left\|f_{(n-s) a_{n}}\right\|=1$ for each $n$ and $s$ while $\left\|a_{s} e_{0}\right\|=a_{s}>1$, this contradicts (2).

Remark. The statement of the theorem remains valid if we consider an equivalent norm on $\ell_{1}$. Indeed, suppose $\frac{1}{K}\|\cdot\| \leqslant\|\cdot\| \leqslant K\|\cdot\|$. Then $\left\|f_{(n-s) a_{n}}\right\| \leqslant K$ for each $n$ and $s$, but since $\lim _{n \rightarrow \infty} a_{n}=\infty$, we can choose $a_{s}$ in (2) so that $\left\|a_{s} e_{0}\right\|>K$.

\section{AN ADJOINT OPERATOR WITH INVARIANT SUBSPACES OF THE FORM $D^{-1} T D$}

Define a sequence of positive reals $\left(d_{i}\right)$ as follows:

$$
d_{i}= \begin{cases}\frac{1}{r} & \text { if } r a_{m} \leqslant i \leqslant r a_{m}+v_{m-r} \text { for some } 0<r \leqslant m \\ 1 & \text { otherwise. }\end{cases}
$$

Let $D$ be the diagonal operator with diagonal $\left(d_{i}\right)$, that is, $D f_{i}=d_{i} f_{i}$ for every $i$. Define $S=D^{-1} T D$. Clearly, $S$ is defined on $F$. Once we write $S$ in matrix form it will be clear that it is bounded on $F$ and, therefore, can be extended to $\ell_{1}$. Let $\hat{e}_{i}=D^{-1} e_{i}$, in particular $\hat{e}_{0}=e_{0}$. Then $S \hat{e}_{i}=D^{-1} T e_{i}=\hat{e}_{i+1}$, so that the sequence $\left(\hat{e}_{i}\right)$ is the orbit of $e_{0}$ under $S$.

Next, we examine Definition 2.1 to represent the $f_{i}$ 's in terms of $\hat{e}_{i}$ 's.

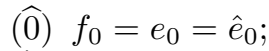

$(\widehat{\mathrm{A}})$ if $i$ satisfies $i \in\left[0, v_{n-r}\right]+r a_{n}$ for some $0<r \leqslant n$, then

$$
f_{i}=d_{i} D^{-1} f_{i}=d_{i} D^{-1}\left(a_{n-r}\left(e_{i}-e_{i-r a_{n}}\right)\right)=\frac{a_{n-r}}{r}\left(\hat{e}_{i}-\hat{e}_{i-r a_{n}}\right) ;
$$

$(\widehat{\mathrm{B}})$ if integers $r, n$, and $i$ satisfy $1 \leqslant r<n, i \in\left(r a_{n}+v_{n-r},(r+1) a_{n}\right)$, (respectively, $1 \leqslant n, i \in\left(v_{n-1}, a_{n}\right)$ ), then

$$
\left.f_{i}=d_{i} D^{-1} f_{i}=2^{(h-i) / \sqrt{a_{n}}} \hat{e}_{i} \text {, where } h=\left(r+\frac{1}{2}\right) a_{n} \text { (respectively, } h=\frac{1}{2} a_{n}\right) \text {; }
$$


$(\widehat{\mathrm{C}})$ if integers $r, n$, and $i$ satisfy $1 \leqslant r \leqslant n, i \in\left[r\left(a_{n}+b_{n}\right), n a_{n}+r b_{n}\right]$, then

$$
f_{i}=d_{i} D^{-1} f_{i}=\hat{e}_{i}-b_{n} \hat{e}_{i-b_{n}} ;
$$

$(\widehat{\mathrm{D}})$ if integers $r, n$, and $i$ satisfy $0 \leqslant r<n, i \in\left(n a_{n}+r b_{n},(r+1)\left(a_{n}+b_{n}\right)\right)$, then

$$
f_{i}=d_{i} D^{-1} f_{i}=2^{(h-i) / \sqrt{b_{n}}} \hat{e}_{i}, \text { where } h=\left(r+\frac{1}{2}\right) b_{n} .
$$

We see that it differs from Definition 2.1 only in case $(\widehat{\mathrm{A}})$. Now we can actually write the matrix of $S$ :

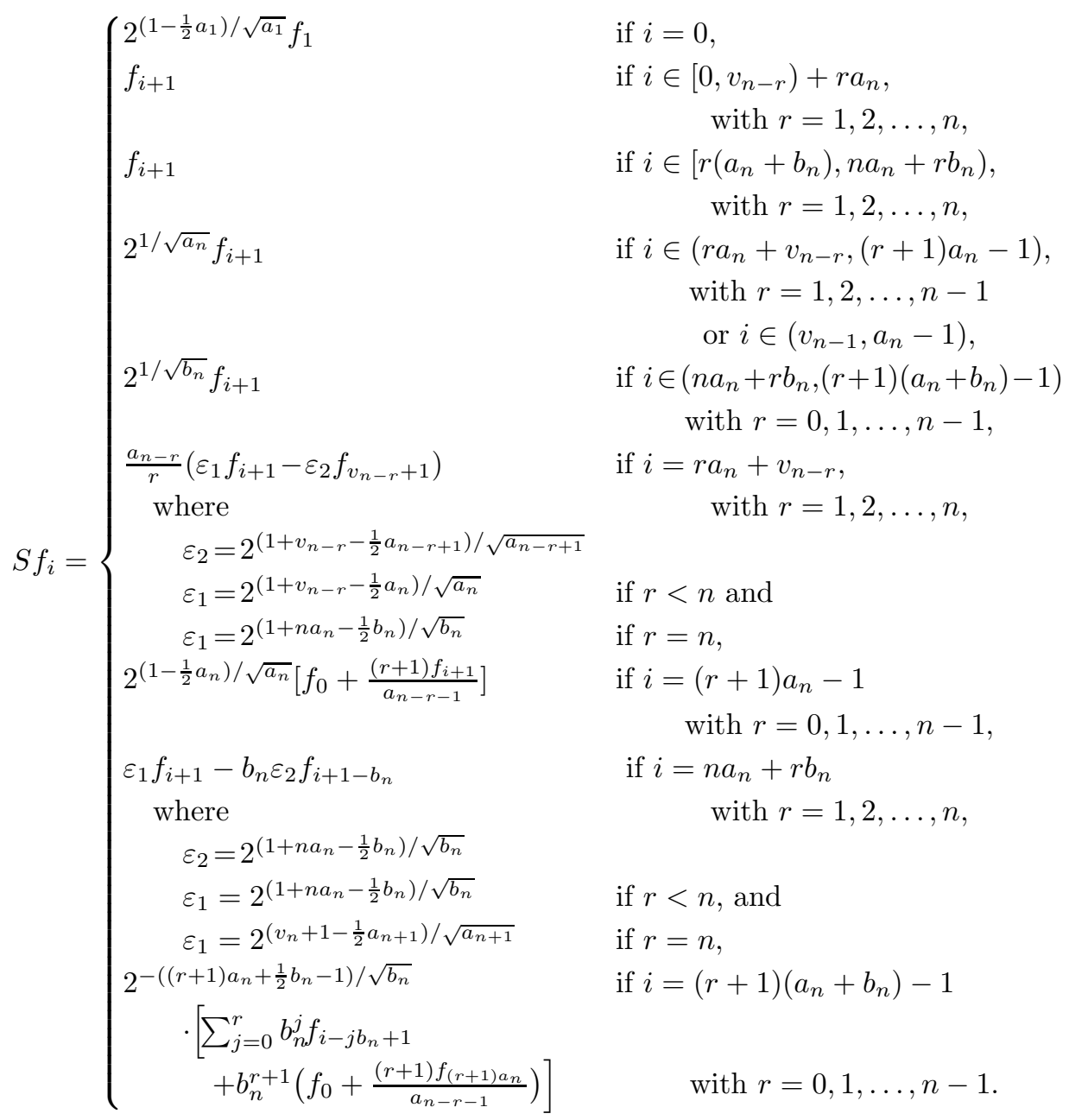

Inspecting the matrix line by line we observe that, assuming $\left(a_{n}\right)$ and $\left(b_{n}\right)$ are increasing sufficiently rapidly, it follows that $\|S\| \leqslant 2$. Again by inspecting each line of the matrix, we deduce that if $f_{j}^{*}$ is the $j$-th coordinate functional on $\ell_{1}$, $j \geqslant 0$, it follows that $\lim _{i \rightarrow \infty} f_{j}^{*}\left(S\left(f_{i}\right)\right)=0$. In other words, the rows of the matrix converge to zero. Therefore $S$ is the adjoint of a linear bounded operator on $c_{0}$.

Theorem 3.1. S has a non-trivial closed invariant subspace. 
We shall show that $S$ has an invariant subspace by producing a vector $x_{\infty}$ such that the linear span of the orbit of $x_{\infty}$ stays away from $e_{0}$, hence its closure is a non-trivial $S$-invariant subspace.

We will introduce the following notations.

First we choose two sequences of positive integers $\left(m_{i}\right)$ and $\left(r_{i}\right)$ as follows. Let $m_{0} \geqslant 2$ be arbitrary, put $r_{0}=1$. Once $m_{i}$ and $r_{i}$ are defined, choose $r_{i+1} \in \mathbb{N}$ so that

$$
r_{i+1} \in\left[a_{m_{i}-1} \cdot \max _{\ell \leqslant v_{m_{i}-1}}\left\|\hat{e}_{\ell}\right\|, 1+a_{m_{i}-1} \cdot \max _{\ell \leqslant v_{m_{i}-1}}\left\|\hat{e}_{\ell}\right\|\right]
$$

and let

$$
m_{i+1}=m_{i}+r_{i+1}
$$

Define an increasing sequence $\left(j_{i}\right)$ of positive integers inductively: pick any

$$
j_{0} \in\left[r_{0} a_{m_{0}}, r_{0} a_{m_{0}}+v_{m_{0}-r_{0}}\right],
$$

and once $j_{i}$ is defined, put

$$
j_{i+1}=j_{i}+r_{i} b_{m_{i}}+r_{i+1} a_{m_{i+1}} .
$$

Finally, for each $i \geqslant 0$ define

$$
\begin{aligned}
\text { (8) } p_{i} & =\prod_{k=0}^{i} b_{m_{k}}^{-r_{k}}, \\
\text { (9) } z_{i} & =f_{j_{i}+r_{i} b_{m_{i}}}+b_{m_{i}} f_{j_{i}+\left(r_{i}-1\right) b_{m_{i}}}+\cdots+b_{m_{i}}^{r_{i}-1} f_{j_{i}+b_{m_{i}}}+\frac{r_{i+1} f_{j_{i+1}}}{a_{m_{i}}}, \\
\text { (10) } x_{i} & =p_{i-1} \hat{e}_{j_{i}} .
\end{aligned}
$$

We note the following easy-to-prove properties for our choices.

Proposition 3.2. For each $i \geqslant 0$ the following statements hold:

(a) $j_{i} \in\left[r_{i} a_{m_{i}}, r_{i} a_{m_{i}}+v_{m_{i}-r_{i}}\right]$;

(b) $x_{i+1}=x_{i}+p_{i} z_{i}$, and thus $x_{i}=\hat{e}_{j_{0}}+\sum_{k=0}^{i-1} p_{k} z_{k}$;

(c) if $i$ and $i+\ell$ both belong to $\left[r a_{n}, r a_{n}+v_{n-r}\right]$ or if they both belong to $\left[r\left(a_{n}+b_{n}\right), n a_{n}+r b_{n}\right]$, then $S^{\ell} f_{i}=f_{i+\ell} ;$

(d) if $\ell<m_{i} a_{m_{i}}-j_{i}$, then min supp $S^{\ell} z_{k} \geqslant j_{i}+b_{m_{i}}$ whenever $k \geqslant i$.

Proof. (a) The proof is by induction. For $i=0$ the required inclusion follows from the choice of $j_{0}$, and if this condition holds for $j_{i}$, then

$$
\begin{aligned}
j_{i+1} & =j_{i}+r_{i} b_{m_{i}}+r_{i+1} a_{m_{i+1}} \\
& \in\left[r_{i} a_{m_{i}}+r_{i} b_{m_{i}}+r_{i+1} a_{m_{i+1}}, r_{i} a_{m_{i}}+v_{m_{i}-r_{i}}+r_{i} b_{m_{i}}+r_{i+1} a_{m_{i+1}}\right] \\
& \subseteq\left[r_{i+1} a_{m_{i+1}}, r_{i+1} a_{m_{i+1}}+m_{i}\left(a_{m_{i}}+b_{m_{i}}\right)\right]=\left[r_{i+1} a_{m_{i+1}}, r_{i+1} a_{m_{i+1}}+v_{m_{i}}\right] .
\end{aligned}
$$

(b) First note that by using $(\widehat{\mathrm{D}})$ we obtain for a $i \in\left[r\left(a_{n}+b_{n}\right), n a_{n}+r b_{n}\right]$, with $1 \leqslant r \leqslant n$ in $\mathbb{N}$, that

$$
\begin{aligned}
\hat{e}_{i} & =b_{n} \hat{e}_{i-b_{n}}+f_{i} \\
& =b_{n}^{2} \hat{e}_{i-2 b_{n}}+b_{n} f_{i-b_{n}}+f_{i} \\
& \vdots \\
& =b_{n}^{r} \hat{e}_{i-r b_{n}}+b_{n}^{r-1} f_{i-(r-1) b_{n}}+\ldots+b_{n} f_{i-b_{n}}+f_{i} .
\end{aligned}
$$


Note that $j_{i}+r_{i} b_{m_{i}} \in\left[r_{i}\left(a_{m_{i}}+b_{m_{i}}\right), m_{i} a_{m_{i}}+r_{i} b_{m_{i}}\right]$. By using first $(\widehat{\mathrm{A}})$ and then (11) we obtain

$$
\begin{aligned}
\hat{e}_{j_{i+1}} & =\hat{e}_{j_{i}+r_{i} b_{m_{i}}+r_{i+1} a_{m_{i}}} \\
& =\hat{e}_{j_{i}+r_{i} b_{m_{i}}}+\frac{r_{i+1}}{a_{m_{i}}} f_{j_{i}+r_{i} b_{m_{i}}+r_{i+1} a_{m_{i}}} \\
& =b_{m_{i}}^{r_{i}} \hat{e}_{j_{i}}+b_{m_{i}}^{r_{i}-1} f_{j_{i}+b_{m_{i}}}+\ldots+b_{m_{i}} f_{j_{i}+\left(r_{i}-1\right) b_{m_{i}}}+\frac{r_{i+1}}{a_{m_{i}}} f_{j_{i}+r_{i} b_{m_{i}}+r_{i+1} a_{m_{i}}} \\
& =b_{m_{i}}^{r_{i}} \hat{e}_{j_{i}}+z_{i} .
\end{aligned}
$$

Thus, $x_{i+1}=p_{i} \hat{e}_{j_{i+1}}=p_{i-1} \hat{e}_{j_{i}}+p_{i} z_{i}=x_{i}+p_{i} z_{i}$.

(c) If $i$ and $i+\ell$ are both in $\left[r a_{n}, r a_{n}+v_{n-r}\right]$, it follows from $(\widehat{A})$ that

$$
S^{\ell}\left(f_{i}\right)=\frac{a_{n-r}}{r} S^{\ell}\left(\hat{e}_{i}-\hat{e}_{i-r a_{n}}\right)=\frac{a_{n-r}}{r}\left(\hat{e}_{i+\ell}-\hat{e}_{i-r a_{n}+\ell}\right)=f_{i+\ell} .
$$

The second part of $(\mathrm{c})$ can be deduced in a similar way using $(\widehat{\mathrm{C}})$.

(d) First note that for $k \geqslant i$ it follows that (recall that $m_{k} \geqslant m_{0} \geqslant 2$ )

$$
m_{k} a_{m_{k}}-j_{k}>\left(m_{k}-r_{k}-1\right) a_{m_{k}}=\left(m_{k-1}-1\right) a_{m_{k}} \geqslant m_{k-1} a_{m_{k-1}}-j_{k-1} .
$$

We can therefore assume that $k=i$. Furthermore, note that for any $1 \leqslant r \leqslant r_{i}$ it follows that

$$
r\left(a_{m_{i}}+b_{m_{i}}\right) \leqslant j_{i}+r b_{m_{i}} \leqslant j_{i}+r b_{m_{i}}+\ell \leqslant m_{i} a_{m_{i}}+r b_{m_{i}}
$$

and

$$
\begin{aligned}
r_{i+1} a_{m_{i+1}} & \leqslant j_{i+1} \leqslant j_{i+1}+\ell \leqslant j_{i+1}+m_{i} a_{m_{i}}-j_{i} \\
& =r_{i+1} a_{m_{i+1}}+r_{i} b_{m_{i}}+m_{i} a_{m_{i}} \\
& \leqslant r_{i+1} a_{m_{i+1}}+v_{m_{i}} \\
& =r_{i+1} a_{m_{i+1}}+v_{m_{i+1}-r_{i+1}} .
\end{aligned}
$$

Therefore the claim follows from the definition of $z_{i}$, (9) and part (c).

Notice that

$$
\left\|z_{i}\right\|=1+b_{m_{i}}+b_{m_{i}}^{2}+\cdots+b_{m_{i}}^{r_{i}-1}+\frac{r_{i+1}}{a_{m_{i}}} \leqslant m_{i} b_{m_{i}}^{r_{i}-1}+\frac{r_{i+1}}{a_{m_{i}}} .
$$

Further, since $p_{i} \leqslant \frac{1}{b_{m_{i}}^{r_{i}}}$, we have

$$
\left\|p_{i} z_{i}\right\| \leqslant \frac{m_{i}}{b_{m_{i}}}+\frac{r_{i+1}}{a_{m_{i}} b_{m_{i}}^{r_{i}}}
$$

The series $\sum_{i=0}^{\infty} \frac{m_{i}}{b_{m_{i}}}$ converges because $\left(b_{i}\right)$ increases sufficiently rapidly. Secondly, it follows from the definition of $\left(r_{i}\right)$ that

$$
a_{m_{i}}^{-1} r_{i+1} \leqslant a_{m_{i}}^{-1}\left[1+a_{m_{i}-1} \cdot \max _{\ell \leqslant v_{m_{i}-1}}\left\|\hat{e}_{\ell}\right\|\right] .
$$

Thus, again since $\left(b_{i}\right)$ is increasing fast enough, it follows that the series

$$
\sum_{i=0}^{\infty} \frac{r_{i+1}}{a_{m_{i}} b_{m_{i}}^{r_{i}}}
$$

converges. Therefore the $\sum_{i=0}^{\infty} p_{i} z_{i}$ converges, and the following definition is justified.

Definition 3.3. Define $x_{\infty}=\lim _{i} x_{i}=\lim _{i} p_{i-1} \hat{e}_{j_{i}}=\hat{e}_{j_{0}}+\sum_{i=0}^{\infty} p_{i} z_{i}$. 
Now we can state and prove the key result for proving Theorem 3.1

Lemma 3.4. There exists a constant $C>0$ such that $\operatorname{dist}\left(y, e_{0}\right) \geqslant C$ for every $i$ and every vector of the form $y=\sum_{j=j_{i}}^{m_{i} a_{m_{i}}} \gamma_{j} \hat{e}_{j}$.

Proof. Let $C=\inf \left\{\operatorname{dist}\left(y, e_{0}\right) \mid y=\sum_{j=j_{0}}^{m_{0} a_{m_{0}}} \gamma_{j} \hat{e}_{j}\right\}$. Since the infimum is taken over a finite-dimensional set, it must be attained at some $y_{0}$. However since all $\hat{e}_{j}$ are linear independent, it follows that $C=\operatorname{dist}\left(y_{0}, e_{0}\right)>0$.

We shall prove the statement of the lemma by induction on $i$. The way we defined $C$ guarantees that the base of the induction holds. Suppose $y=\sum_{j=j_{i}}^{m_{i} a_{m_{i}}} \gamma_{j} \hat{e}_{j}$. Write $y=y_{1}+y_{2}+y_{3}$, where

$$
y_{1}=\sum_{j=j_{i}}^{r_{i} a_{m_{i}}+v_{m_{i-1}}} \gamma_{j} \hat{e}_{j}, \quad y_{2}=\sum_{r=r_{i}+1}^{m_{i}} \sum_{j=r a_{m_{i}}}^{r a_{m_{i}}+v_{m_{i}-r}} \gamma_{j} \hat{e}_{j}, \quad \text { and } \quad y_{3}=\sum_{r=r_{i}}^{m_{i}-1} \sum_{j=r a_{m_{i}}+v_{m_{i}}-r+1}^{(r+1) a_{m_{i}}-1} \gamma_{j} \hat{e}_{j} .
$$

Notice that by $(\widehat{\mathrm{B}})$

$$
y_{3}=\sum_{r=r_{i}}^{m_{i}-1} \sum_{j=r a_{m_{i}}+v_{m_{i}-r}+1}^{(r+1) a_{m_{i}}-1} \gamma_{j} 2^{-\left(r+\frac{1}{2}-j\right) / \sqrt{a_{m_{i}}}} f_{j},
$$

so that $\operatorname{supp} y_{3} \subseteq \bigcup_{r=r_{i}}^{m_{i}-1}\left(r a_{m_{i}}+v_{m_{i}-r},(r+1) a_{m_{i}}\right)$. Furthermore, using $(\widehat{\mathrm{A}})$, we write $y_{2}=y_{2}^{\prime}+y_{2}^{\prime \prime}$ where

$$
\begin{gathered}
y_{2}^{\prime}=\sum_{r=r_{i}+1}^{m_{i}} \sum_{j=r a_{m_{i}}}^{r a_{m_{i}}+v_{m_{i}-r}} \gamma_{j} \hat{e}_{j-r a_{m_{i}}}=\sum_{r=r_{i}+1}^{m_{i}} \sum_{j=0}^{v_{m_{i}-r}} \gamma_{j+r a_{m_{i}}} \hat{e}_{j} \\
\text { and } y_{2}^{\prime \prime}=\sum_{r=r_{i}+1}^{m_{i}} \sum_{j=r a_{m_{i}}}^{r a_{m_{i}}+v_{m_{i}-r}} \frac{\gamma_{j} r}{a_{m_{i}-r}} f_{j} .
\end{gathered}
$$

Therefore,

$$
\operatorname{supp}\left(y_{1}+y_{2}\right) \subseteq\left[0, r_{i} a_{m_{i}}+v_{m_{i-1}}\right] \cup \bigcup_{r=r_{i}+1}^{m_{i}}\left[r a_{m_{i}}, r a_{m_{i}}+v_{m_{i}-r_{i}}\right] .
$$

One observes that the vectors $y_{1}+y_{2}$ and $y_{3}$ have disjoint supports; it follows that $\operatorname{dist}\left(y, e_{0}\right) \geqslant \operatorname{dist}\left(y_{1}+y_{2}, e_{0}\right)$.

Furthermore,

$$
\left\|y_{2}^{\prime}\right\|=\left\|\sum_{r=r_{i}+1}^{m_{i}} \sum_{j=r a_{m_{i}}}^{r a_{m_{i}}+v_{m_{i}-r}} \gamma_{j} \hat{e}_{j-r a_{m_{i}}}\right\| \leqslant \sum_{r=r_{i}+1}^{m_{i}} \sum_{j=r a_{m_{i}}}^{r a_{m_{i}}+v_{m_{i}-r}}\left|\gamma_{j}\right| \cdot \max _{k \leqslant v_{m_{i-1}-1}}\left\|\hat{e}_{k}\right\| .
$$

By choice of $\left(r_{i}\right)$ (44), we have $\max _{k \leqslant v_{m_{i-1}-1}}\left\|\hat{e}_{k}\right\| \leqslant \frac{r_{i}}{a_{m_{i}-r_{i}-1}} \leqslant \frac{r}{a_{m_{i}-r}}$ when $r_{i}<r \leqslant m_{i}$. This yields

$$
\left\|y_{2}^{\prime}\right\| \leqslant\left\|\sum_{r=r_{i}+1}^{m_{i}} \sum_{j=r a_{m_{i}}}^{r a_{m_{i}}+v_{m_{i}-r}} \frac{\gamma_{j} r}{a_{m_{i}-r}} f_{j}\right\|=\left\|y_{2}^{\prime \prime}\right\|
$$


Since the support of $y_{2}^{\prime \prime}$ is disjoint from that of $y_{1}+y_{2}^{\prime}$ and doesn't contain 0 , we have

$$
\begin{aligned}
\operatorname{dist}\left(y_{1}, e_{0}\right) & \leqslant \operatorname{dist}\left(y_{1}+y_{2}^{\prime}, e_{0}\right)+\left\|y_{2}^{\prime}\right\| \\
& =\operatorname{dist}\left(y_{1}+y_{2}^{\prime}+y_{2}^{\prime \prime}, e_{0}\right)-\left\|y_{2}^{\prime \prime}\right\|+\left\|y_{2}^{\prime}\right\| \\
& \leqslant \operatorname{dist}\left(y_{1}+y_{2}, e_{0}\right) \leqslant \operatorname{dist}\left(y, e_{0}\right) .
\end{aligned}
$$

It is left to show that $\operatorname{dist}\left(y_{1}, e_{0}\right) \geqslant C$. Since $j_{i} \geqslant r_{i} a_{m_{i}}$, it follows from $(\widehat{\mathrm{A}})$ that $y_{1}=y_{1}^{\prime}+y_{1}^{\prime \prime}$ where

$$
y_{1}^{\prime}=\sum_{j=j_{i}}^{r_{i} a_{m_{i}}+v_{m_{i-1}}} \gamma_{j} \hat{e}_{j-r_{i} a_{m_{i}}} \text { and } y_{1}^{\prime \prime}=\sum_{j=j_{i}}^{r_{i} a_{m_{i}}+v_{m_{i-1}}} \frac{\gamma_{j} r}{a_{m_{i}-r_{i}}} f_{j} .
$$

Since $j_{i}=j_{i-1}+r_{i-1} b_{m_{i-1}}+r_{i} a_{m_{i}}$, we have $y_{1}^{\prime}=\sum_{j=j_{i-1}+r_{i-1} b_{m_{i-1}}}^{v_{m_{i-1}}} \beta_{j} \hat{e}_{j}$, where $\beta_{j}=\gamma_{j+r_{i} a_{m_{i}}}$. In particular this means that $\operatorname{supp} y_{1}^{\prime} \subseteq\left[0, v_{m_{i-1}}\right]$, while min supp $y_{1}^{\prime \prime}$ $\geqslant j_{i} \geqslant r_{i} a_{m_{i}}$. Thus, the supports are disjoint, which yields $\operatorname{dist}\left(y_{1}, e_{0}\right) \geqslant \operatorname{dist}\left(y_{1}^{\prime}, e_{0}\right)$.

Split the index set of $y_{1}^{\prime}$ into two disjoint subsets: let

$$
\begin{aligned}
& A=\left[j_{i-1}+r_{i-1} b_{m_{i-1}}, v_{m_{i-1}}\right] \cap \bigcup_{r=r_{i-1}}^{m_{i-1}}\left(m_{i-1} a_{m_{i-1}}+r b_{m_{i-1}},(r+1)\left(a_{m_{i-1}}+b_{m_{i-1}}\right)\right), \\
& B=\left[j_{i-1}+r_{i-1} b_{m_{i-1}}, v_{m_{i-1}}\right] \cap \bigcup_{r=r_{i-1}}^{m_{i-1}}\left[r\left(a_{m_{i-1}}+b_{m_{i-1}}\right), m_{i-1} a_{m_{i-1}}+r b_{m_{i-1}}\right] .
\end{aligned}
$$

Write $y_{1}^{\prime}=z_{a}+z_{b}$ where $z_{a}=\sum_{j \in A} \beta_{j} \hat{e}_{j}$ and $z_{b}=\sum_{j \in B} \beta_{j} \hat{e}_{j}$. For $j \in A$ we have $\hat{e}_{j}=2^{\left((r+1 / 2) b_{m_{i-1}}-j\right) / \sqrt{b_{m_{i-1}}}} f_{j}$, so that $\operatorname{supp} z_{a} \subseteq A$. In view of (11) we can write $z_{b}=z_{b}^{\prime}+z_{b}^{\prime \prime}$, where

$$
z_{b}^{\prime}=\sum_{j \in B} \sum_{k=0}^{r-1} \beta_{j} b_{m_{i-1}}^{k} f_{j-k b_{m_{i-1}}} \quad \text { and } \quad z_{b}^{\prime \prime}=\sum_{j \in B} \beta_{j} b_{m_{i-1}}^{r} \hat{e}_{j-r b_{m_{i-1}}} .
$$

We first note that $\operatorname{supp} z_{b}^{\prime} \subseteq B$ and determine the support of $z_{b}^{\prime \prime}$ as follows. If $j \in B$, then $j \geqslant j_{i-1}+r_{i-1} b_{m_{i-1}}$ and $j \in\left[r\left(a_{m_{i-1}}+b_{m_{i-1}}\right), m_{i-1} a_{m_{i-1}}+r b_{m_{i-1}}\right]$ for some $r \in\left[r_{i-1}, m_{i-1}\right]$. If $r=r_{i-1}$, then $j-r b_{m_{i-1}} \geqslant j_{i-1}$. If $r>r_{i-1}$, then $j-r b_{m_{i-1}} \geqslant r a_{m_{i-1}}>r_{i-1} a_{m_{i-1}}+v_{m_{i-2}} \geqslant j_{i-1}$ by (17). We see that $z_{b}^{\prime \prime}$ is a linear combination of $\hat{e}_{j}$ 's with $j_{i-1} \leqslant j \leqslant m_{i-1} a_{m_{i-1}}$. Hence its support is contained in $\left[0, m_{i-1} a_{m_{i-1}}\right]$ and, therefore, is disjoint from that of $z_{a}$ and $z_{b}^{\prime}$. It follows that $\operatorname{dist}\left(y, e_{0}\right) \geqslant \operatorname{dist}\left(y_{1}^{\prime}, e_{0}\right) \geqslant \operatorname{dist}\left(z_{b}^{\prime \prime}, e_{0}\right)$. Finally, $\operatorname{dist}\left(z_{b}^{\prime \prime}, e_{0}\right) \geqslant C$ by the induction hypothesis.

Proof of Theorem [3.1. We will prove that the linear span of the orbit of $x_{\infty}$ under $S$ is at least distance $C$ from $e_{0}$, hence its closure is a non-trivial invariant subspace for $S$. Consider a linear combination $\sum_{\ell=0}^{N} \alpha_{\ell} S^{\ell} x_{\infty}$. It follows from (7) that the sequence $\left(m_{i} a_{m_{i}}-j_{i}\right)$ is unbounded, so that $N<m_{i} a_{m_{i}}-j_{i}$ for some $i \geqslant 0$. Recall that $x_{\infty}=x_{i}+\sum_{k=i}^{\infty} p_{k} z_{k}$; then

$$
\sum_{\ell=0}^{N} \alpha_{\ell} S^{\ell} x_{\infty}=\sum_{s=0}^{N} \alpha_{\ell} S^{\ell} x_{i}+\sum_{\ell=0}^{N} \sum_{k=i}^{\infty} \alpha_{\ell} S^{\ell}\left(p_{k} z_{k}\right)
$$


Notice that the two sums have disjoint supports, and the support of the second one does not contain 0 . Indeed, since $x_{i}=p_{i-1} \hat{e}_{j_{i}}$, then $S^{\ell} x_{i}=p_{i-1} \hat{e}_{j_{i}+\ell}$ for $\ell=1, \ldots, N$. Furthermore,

$$
j_{i} \leqslant j_{i}+\ell \leqslant j_{i}+N<j_{i}+\left(m_{i} a_{m_{i}}-j_{i}\right)=m_{i} a_{m_{i}} .
$$

It follows that $\sum_{\ell=0}^{N} S^{\ell} x_{i}$ is a linear combination of $\hat{e}_{j}$ 's with $j_{i} \leqslant j \leqslant m_{i} a_{m_{i}}$. In particular, its support is contained in $\left[0, m_{i} a_{m_{i}}\right]$. On the other hand, Proposition 3.2 (d) implies that

$$
\min \operatorname{supp}\left(\sum_{\ell=0}^{N} \sum_{k=i}^{\infty} S^{\ell}\left(p_{k} z_{k}\right)\right) \geqslant j_{i}+b_{m_{i}} .
$$

Therefore, by Lemma 3.4

$$
\operatorname{dist}\left(\sum_{\ell=0}^{N} S^{\ell} x_{\infty}, e_{0}\right) \geqslant \operatorname{dist}\left(\sum_{\ell=0}^{N} S^{\ell} x_{i}, e_{0}\right) \geqslant C .
$$

\section{REFERENCES}

[AAB98] Y. A. Abramovich, C. D. Aliprantis, and O. Burkinshaw. The invariant subspace problem: Some recent advances. Rend. Inst. Mat. Univ. Trieste, XXIX Supplemento:3-79, 1998. MR 2000f: 47062

[Enf76] P. Enflo. On the invariant subspace problem in Banach spaces. In Séminaire MaureySchwartz (1975-1976) Espaces L ${ }^{p}$, applications radonifiantes et géométrie des espaces de Banach, Exp. Nos. 14-15, pages 1-7. Centre Math., École Polytech., Palaiseau, 1976. MR 57:13530

[Enf87] P. Enflo. On the invariant subspace problem in Banach spaces. Acta Math., 158: 213313, 1987. MR 88j:47006

[Read85] C. J. Read. A solution to the invariant subspace problem on the space $l_{1}$. Bull. London Math. Soc., 17(4):305-317, 1985. MR 87e:47013

[Read86] C. J. Read. A short proof concerning the invariant subspace problem. J. London Math. Soc. (2), 34(2):335-348, 1986. MR 87m:47020

[RR73] H. Radjavi and P. Rosenthal. Invariant subspaces. Springer-Verlag, New York, 1973. Ergebnisse der Mathematik und ihrer Grenzgebiete, Band 77. MR 51:3924

[Sz-NF68] B. Sz.-Nagy and C. Foiaş. Vecteurs cycliques et quasi-affinité. Studia Math. 31: 35-42, 1968. MR 38:5050

Department of Mathematics, Texas A\&M University, College Station, Texas 77843

E-mail address: schlump@math.tamu.edu

Department of Mathematics, University of Alberta, Edmonton, Alberta, Canada T6G 2G1

E-mail address: vtroitsky@math.ualberta.ca 